\title{
Letter to the Editor/Leserbrief zu dem Beitrag von C. D. Reimers, M. Wolf, T. Kretschmer und J. Röther „Schwieriger magnetresonanztomografischer Nachweis eines Hämangioms des N. facialis - ein Fallbericht"
}

Die Autoren des Fallberichtes beschreiben den Fall einer offensichtlich über mehrere Jahre verzögerten Diagnose einer vaskulären Anomalie der Schädelbasis. In einer dünnschichtigen MRT-Untersuchung, die für die Fragestellung sicherlich Standard ist, konnte die Pathologie entsprechend detektiert werden, gefolgt von einer mikrochirurgischen Resektion mit Rekonstruktion des assoziierten Nervus facialis, bedauerlicherweise mit nur moderatem klinischen Ergebnis.

Raumforderungen der Schädelbasis, insbesondere geringer Größe stellen auch für geübte Kopf-Hals Radiologen eine Herausforderung da, da die Anzahl an möglichen Differenzialdiagnosen groß ist und gleichermaßen die klinische Symptomatik durch Affektion der verschiedensten neuralen Strukturen komplex sein kann. Auf den dünnschichtigen MRT-Aufnahmen zeigt sich in der T2-gewichteten CISS-Sequenz eine hyperintense Läsion angrenzend an den N. facialis, innerhalb dieser sich multiple hypodense rundliche Strukturen abgrenzen lassen. Ob es sich dabei um orthogonal getroffene Nervenfasern handelt wird in dem Bericht nicht genauer erläutert. In der T1-gewichteten Sequenz erscheint das T2-hyperintense Areal isointens zum angrenzenden Cortex, nach Kontrastmittelgabe zeigt sich eine relative homogene Anreicherung der Läsion, wobei die nodulären Strukturen weiterhin hypointens zur Darstellung kommen. Intraoperativ zeigt sich eine livide Raumforderung angrenzend an das Felsenbein.

In Zusammenschau des radiologischen Befundes mit der Histologie, die bedauerlicherweise nicht gezeigt wird, wird der Befund eines Hämangioms des N. facialis gestellt.

Diese Diagnose ist falsch und gleichzeitig muss auf eine korrekte Terminologie der beschriebenen Pathologien hingewiesen werden.

Vaskuläre Anomalien lassen sich gemäß den Richtlinien der International Society for the Study of Vascular Anomalies ISSVA einteilen in Gefäßtumore und Gefäßmal- formationen, die detaillierte Klassifikation ist über www.issva.org abrufbar.

Infantile Hämangiome sind in den allermeisten Fällen unmittelbar nach der Geburt neu auftretende, schnell wachsende und spontan innerhalb der ersten zwei Lebensjahre sich zurückbildende vaskuläre Tumoren des Kindes- respektive Neugeborenenalters. Die sehr seltenen Hämangiome, die bei Geburt bereits vorhanden sind, die dann aber in den häufigsten Fällen innerhalb der ersten Lebensjahre regredient sind, werden Rapid Involuting Congenital Hemangioma (RICH) genannt. Nur sehr selten bilden sich Hämangiome nicht zurück und können sogar progredient sein, dann spricht man von Non-Involuting Congenital Hemangioma (NICH) (Uller W et al. Interventionelle Radiologie Scan 2013; 3: 235-246).

Hämangiome des Erwachsenenalters sind dagegen eine absolute Rarität mit distinkter Histologie. Anders vaskuläre Malformationen: diese sind bei Geburt oft klinisch noch nicht nachweisbar und werden erst im Verlauf der Adoleszenz oder später progredient, bis zur klinischen Manifestation. Vaskuläre Malformationen fallen somit oftmals erst im Erwachsenalter auf, gleichwohl es sich dabei um einen frühen Defekt in der Embryogenese handelt. Ebenfalls bilden sich vaskuläre Malformationen nie von selbst zurück. Die Unterscheidung von Gefäßtumoren/Hämangiomen und Gefäßmalformationen ist dabei keinesfalls eine rein semantische:

Während Hämangiomen als Pathophysiologie eine Metaplasie mit endothelialer Proliferation zugrunde liegt, sind Vaskuläre Malformationen durch eine Dysplasie mit gestörter vaskulärer Morphogenese gekennzeichnet: Den Gefäßwänden venöser Malformationen fehlen glatte Muskelzellen, sodass es zu abnormen Aussackungen dieser Gefäße kommt. Liegen diese Aussackungen in der Nähe neuraler Strukturen kann es hier zu Affektion des Nerven bzw. bei längerfristiger Kompression sogar zur Druckatrophie mit klinischen Lähmungserscheinungen kommen.
Die Unterscheidung in Gefäßmalformationen und vaskuläre Tumore ist vor allem in Bezug auf das therapeutische Prozedere relevant. Während Gefäßmalformationen bevorzugt embolisiert (Fast-flow-Malformationen) oder sklerosiert (Slow-flowMalformationen) werden, werden Infantile Hämangiome medikamentös (Propranolol) oder kryo-/laserchirurgisch therapiert.

Die somit wichtige differenzialdiagnostische Unterscheidung von Gefäßmalformationen und Gefäßtumoren/Hämangiomen wurde in dem von Reimers et al. präsentierten Fall nicht getroffen und die Schilderung des Befundes als „Kavernöse Malformation (Hämangiom)" folgt nicht der gültigen Klassifikation, sondern stellt eine contradictio in eo ipso dar. Der Befund eines Hämangioms im Alter von 48 Jahren ist praktisch auszuschließen. Leider hat sich die korrekte Terminologie vaskulärer Anomalien auch in histopathologischen Befunden oft noch nicht durchgesetzt. Die histopathologische Differenzialdiagnose von vaskulären Tumoren im Vergleich zu Gefäßmalformationen ist gleichermaßen nicht einfach. Die früher häufig als pathognomonisch für ein Hämangiom beschriebene Positivität für GLUT-1 ist dabei ebenfalls nicht mehr haltbar, da nachgewiesenermaßen auch Malformationen positiv für den Glucosetransporter GLUT-1 sein können (de Oliviera DH. J Oral Pathol Med 2014; 43: 76-80).

Ob es sich bei dem Patienten tatsächlich um eine venöse Gefäßmalformation handelt, kann hier abschließend, vor allem ohne die Vorlage der Histologie nicht beurteilt werden, ist aber von der Bildgebung her sehr wahrscheinlich. Der magnetresonanztomografische Befund einer hyperintense Läsion in T2, die nach Gadoliniumgabe „zuläuft“ und homogen anreichert ist mit dem Befund einer venösen Malformation sehr gut vereinbar. Auch der geschilderte undulierende klinische Verlauf, mit rezidivierender Symptomatik und zwischenzeitlicher klinischer Besserung passt gut zu einer venösen Malformation: durch den langsamen Blutfluss inner- 
halb der Malformation kommt es typischerweise zu lokalen Thrombosen, die sich aseptisch entzünden können und als rezidivierende Thrombophlebitiden die venöse Malformation repetitiv schmerzhaft werden lassen. Die endgültige Diagnose des hier dargelegten Falls ist damit weiter unklar, die Diagnose einer venösen Gefäßmalformation (low-flow) erscheint aber wahrscheinlich.

Die von den Autoren dargelegte Schlussfolgerung „Therapie der Wahl ist die operative Entfernung “ ist damit ebenfalls kritisch zu hinterfragen. Mutmaßlich wäre eine perkutane Sklerosierung des Befundes technisch schwierig gewesen, eine Verallgemeinerung der therapeutischen Strategie in Bezug auf eine chirurgische Resektion ist jedoch nicht zeitgemäß.

Vaskuläre Anomalien erfordern eine dedizierte interdisziplinäre Diagnostik und

\section{Antwort der Autoren}

Der Autor nimmt zu unserem Fallbericht über ein kavernöses Kavernom des N. facialis Stellung. Dabei wird insbesondere die Diagnose des kavernösen Hämangioms aus theoretischen Erwägungen bezweifelt. Tatsächlich wurde das histopathologische Operationspräparat jedoch als regressiv verändertes kavernöses Hämangiom befundet. Die Diagnose wurde im Referenzlabor bestätigt.

Bei der Diagnose handelte es sich um einen überraschenden Befund. Präoperativ wurde in erster Linie an ein Neurinom gedacht und daher die Operationsindikation gestellt.

Der Hinweis auf das schlechte Outcome ist zu relativieren, denn zum Einen ist die
Therapie, die in ausgewiesenen Zentren erfolgen sollte. Die Radiologie spielt hierbei eine zentrale Rolle sowohl in der Diagnostik als auch der Therapie und sollte auf eine korrekte terminologische Befundbeschreibung achten um die Patienten, häufig Kinder und junge Erwachsende, auf die richtige Therapiestrategie zu lenken.

\section{Autoren}

Moritz Wildgruber' ${ }^{1}$, Walter A. Wohlgemuth ${ }^{2}$, Michael Köhler ${ }^{1}$

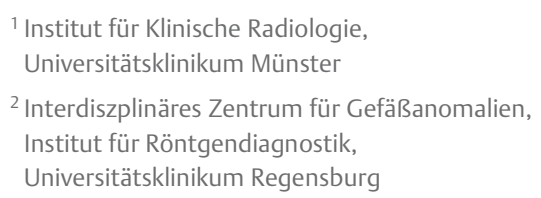

Korrespondenzadresse

Prof. Dr. Moritz Wildgruber Institut für Klinische Radiologie, Universitätsklinikum Münster Albert Schweitzer Campus 1 48149 Münster

Germany

moritz.wildgruber@ukmuenster.de

Bibliografie

DOI http://dx.doi.org/10.1055/s-0042-122148 Online-Publikation: 2017 | Fortschr Röntgenstr 2017; 189: 257-258 @ Georg Thieme Verlag KG Stuttgart · New York

ISSN 1438-9029

Fazialisfunktion nicht komplett aufgehoben, zum Anderen ist der Nachbeobachtungszeitraum noch viel zu kurz, um ein Statement über die letztlich erreichte Funktion abzugeben.

Der Auffassung, dass sich vaskuläre Malformationen von selbst zurückbilden wird widersprochen. Vielmehr ist eine mikrochirurgische Operation Mittel der Wahl (Rotondo M, Natale M, D'Avanzo R et al. Clin Neurol Neurosurg. 2014; 126: 162168). Auch dem Vorschlag einer interventionellen Behandlung wird widersprochen: Eine kavernöse Malformation ist im weitesten Sinne eine multipel septierte, gefäßwandartig ummantelte Raumforderung, die innerhalb ihrer Kavernen Blut und Blutabbauprodukte unterschiedlichen Alters enthält. Sie ist nicht an das Hauptgefäßsystem angeschlossen und kann alleine schon deswegen nicht endovaskulär behandelt werden. Zusätzlich liegt auch kein Hohlraum vor, sondern multiple Kavernen, die teilweise auch bindegewebig und regredient umgewandelt sind.

Prof. Dr. med. C. D. Reimers

MVZ Neurologie

Paracelsus-Klinik Bremen

In der Vahr 65

283239 Bremen

Tel. 0160-8417307

E-Mail c.d.reimers@outlook.de 